\title{
TRANSFER PROPERTIES OF GORENSTEIN HOMOLOGICAL DIMENSION WITH RESPECT TO A SEMIDUALIZING MODULE
}

\author{
ZhenXing Di AND XiaOyan Yang
}

\begin{abstract}
The classes of $G_{C}$ homological modules over commutative ring, where $C$ is a semidualizing module, extend Holm and Jøgensen's notions of $C$-Gorenstein homological modules to the non-Noetherian setting and generalize the classical classes of homological modules and the classes of Gorenstein homological modules within this setting. On the other hand, transfer of homological properties along ring homomorphisms is already a classical field of study. Motivated by the ideas mentioned above, in this article we will investigate the transfer properties of $C$ and $G_{C}$ homological dimension.
\end{abstract}

\section{Introduction}

Unless stated otherwise, all rings in this paper are assumed to be commutative, unital and non-zero; throughout, $R$ and $S$ denote such rings. All modules are unitary. We use abbreviations pd, id and fd for projective, injective and flat dimension of modules. By $P(R), I(R)$ and $F(R)$ we denote the full subcategories of the category of $R$-modules whose objects are modules of finite projective, injective and flat dimension.

Foxby [5], Vasconcelos [13] and Golod [6] independently initiated the study of semidualizing modules (under different names). Over a Noetherian ring $R$, a finitely generated $R$-module $C$ is semidualizing if the natural homothety map $R \rightarrow \operatorname{Hom}_{R}(C, C)$ is an isomorphism and $\operatorname{Ext}_{R}^{\geqslant 1}(C, C)=0$; see Definition 2.3 for a more general definition. In [6] Golod used a semidualizing module $C$ to define totally $C$-reflexive modules and $G_{C}$-dimension for finitely generated modules, which are refinements of projective modules and projective dimension. Several decades later, Holm and Jøgensen [8] extended these notions by introducing $C$-Gorenstein projective and flat modules and corresponding homological dimensions to arbitrary modules over a commutative Noetherian ring. Meanwhile, the notion of $C$-Gorenstein injective modules was also introduced

Received April 10, 2011; Revised August 15, 2011.

2010 Mathematics Subject Classification. 13D05, 13D02, 16E10, 16E30.

Key words and phrases. semidualizing module, ring homomorphism, $C$ homological modules, $G_{C}$ homological modules, localization. 
in [8]. Recently, White [15] has extended the notions of $C$-Gorenstein projective modules and $C$-Gorenstein projective dimension to the non-Noetherian setting. Similar to the proofs in [15], we can also get some results regarding the other two types of module classes; see Remark 2.7(2). On the other hand, transfer of homological properties along ring homomorphisms is already a classical field of study. In [3] Christensen and Holm comprehensively investigated the transfer properties of the classes of Gorenstein homological modules. Affected by [3], in this article, we will discuss the transfer properties of the classes of $C$ and $G_{C}$ homological modules along ring homomorphisms.

In Section 3, we will give some auxiliary propositions and lemmas which play a crucial role in proving our main results of Section 4. Propositions 3.1 and 3.3 contain the transfer properties of the classes of $C$ homological modules along ring homomorphisms.

We will discuss, in Section 4, the transfer properties of the classes of $G_{C}$ homological modules along ring homomorphisms. For instance, assume that $\varphi$ : $R \rightarrow S$ is a homomorphism of rings and $\tilde{C}$ is a semidualizing $S$-module. Then $\tilde{B}$ is a $G_{\tilde{C}}$-flat $S$-module if and only if $\tilde{B} \otimes_{R} F$ is a $G_{\tilde{C}}$-flat $S$-module for any flat $R$-module $F$; see Proposition 4.1, etc. We will also study some properties of the classes of $G_{C}$ homological modules in this section. For instance, the class $\mathcal{G F}_{C}(R)$ is closed under direct limits when $R$ is a coherent ring and $C$ is a semidualizing $R$-module; see Theorem 4.3 , etc. In particular, in the remainder of this section we will focus our attention to the localization of the classes of $G_{C}$ homological modules.

\section{Notions and definitions}

In this section, we mainly recall some necessary notions and definitions. Let $\mathcal{X}=\mathcal{X}(R)$ be a class of $R$-modules.

2.1 Resolutions. For any $R$-module $M$, we recall three types of resolutions.

(1) $[7,1.5]$ A left $\mathcal{X}$-resolution of $M$ is an exact sequence $\mathbb{X}=\cdots \rightarrow X_{1} \rightarrow$ $X_{0} \rightarrow M \rightarrow 0$ with $X_{n} \in \mathcal{X}$ for all $n \geqslant 0$.

(2) $[7,1.5]$ A right $\mathcal{X}$-resolution of $M$ is an exact sequence $\mathbb{X}=0 \rightarrow M \rightarrow$ $X^{0} \rightarrow X^{1} \rightarrow \cdots$ with $X^{n} \in \mathcal{X}$ for all $n \geqslant 0$.

Now let $\mathbb{X}$ be any (left or right) $\mathcal{X}$-resolution of $M$. We say that $\mathbb{X}$ is coproper if the sequence $\operatorname{Hom}_{R}(\mathbb{X}, Y)$ is exact for all $Y \in \mathcal{X}$.

(3) $[15,1.6]$ A degreewise finite projective (resp., free) resolution of $M$ is a left projective (resp., free) resolution $\mathbb{P}$ of $M$ such that each $P_{i}$ is finitely generated projective (resp., free). It is easy to verify that $M$ admits a degreewise finite projective resolution if and only if $M$ admits a degreewise finite free resolution.

2.2 Dimensions. The $\mathcal{X}$-projective dimension of an $R$-module $M$ is defined as $\mathcal{X}-\operatorname{pd}_{R}(M)=\inf \left\{\sup \left\{n \mid X_{n} \neq 0\right\} \mid \mathbb{X}\right.$ is a left $\mathcal{X}$-resolution of $\left.M\right\}$.

Dually, we can also define the $\mathcal{X}$-injective dimension of $M$.

Definition 2.3 ([15, 1.8]). An $R$-module $C$ is semidualizing if 
(1) $C$ admits a degreewise finite projective resolution,

(2) The natural homothety map $R \rightarrow \operatorname{Hom}_{R}(C, C)$ is an isomorphism and

(3) $\operatorname{Ext}_{R}^{\geqslant 1}(C, C)=0$.

A free $R$-module of rank one is semidualizing. If $R$ is Noetherian and admits a dualizing module $D$, then $D$ is semidualizing.

In the following part of this section, let $C$ be a semidualizing $R$-module.

Definition 2.4 ([9, Definition 5.1]). An $R$-module is $C$-projective (resp., $C$ flat) if it has the form $C \otimes_{R} P$ for some projective (resp., flat) $R$-module $P$. An $R$-module is $C$-injective if it has the form $\operatorname{Hom}_{R}(C, I)$ for some injective $R$-module $I$. We set

$$
\begin{aligned}
& \mathcal{P}_{C}(R)=\left\{C \otimes_{R} P \mid P \text { is a projective } R \text {-module }\right\}, \\
& \mathcal{F}_{C}(R)=\left\{C \otimes_{R} F \mid F \text { is a flat } R \text {-module }\right\}, \\
& \mathcal{I}_{C}(R)=\left\{\operatorname{Hom}_{R}(C, I) \mid I \text { is an injective } R \text {-module }\right\} .
\end{aligned}
$$

Remark 2.5. The classes defined above are studied extensively in [9]. From there we know that

(1) The classes $\mathcal{F}_{C}(R)$ and $\mathcal{P}_{C}(R)$ are closed under arbitrary direct sums and summands and if $R$ is coherent, then $\mathcal{F}_{C}(R)$ is also closed under arbitrary direct products.

(2) The class $\mathcal{I}_{C}(R)$ is closed under arbitrary direct products and summands.

Definition 2.6 ([15, Definition 2.1]). An $R$-module $M$ is said to be $G_{C^{-}}$ projective ( $G_{C}$-proj for short) if there exists an exact sequence of $R$-modules

$$
\mathbb{X}=\cdots \longrightarrow P_{1} \longrightarrow P_{0} \longrightarrow C \otimes_{R} P^{0} \longrightarrow C \otimes_{R} P^{1} \longrightarrow \cdots,
$$

where each $P_{i}$ and $P^{i}$ is projective, such that $M \cong \operatorname{Im}\left(P_{0} \rightarrow C \otimes_{R} P^{0}\right)$ and the sequence $\operatorname{Hom}_{R}\left(\mathbb{X}, C \otimes_{R} Q\right)$ is exact for each projective $R$-module $Q$. The exact sequence $\mathbb{X}$ is called a complete $P_{C}$-resolution of $M$.

Dually, an $R$-module $N$ is said to be $G_{C}$-injective ( $G_{C}$-inj for short) if there exists an exact sequence of $R$-modules

$$
\mathbb{Y}=\cdots \longrightarrow \operatorname{Hom}_{R}\left(C, I^{1}\right) \longrightarrow \operatorname{Hom}_{R}\left(C, I^{0}\right) \longrightarrow I_{0} \longrightarrow I_{1} \longrightarrow \cdots,
$$

where each $I_{i}$ and $I^{i}$ is injective, such that $N \cong \operatorname{Im}\left(\operatorname{Hom}_{R}\left(C, I^{0}\right) \rightarrow I_{0}\right)$ and the sequence $\operatorname{Hom}_{R}\left(\operatorname{Hom}_{R}(C, I), \mathbb{Y}\right)$ is exact for each injective $R$-module $I$. The exact sequence $\mathbb{Y}$ is called a complete $I_{C}$-resolution of $N$.

An $R$-module $T$ is said to be $G_{C}$-flat if there exists an exact sequence of $R$-modules

$$
\mathbb{Z}=\cdots \longrightarrow F_{1} \longrightarrow F_{0} \longrightarrow C \otimes_{R} F^{0} \longrightarrow C \otimes_{R} F^{1} \longrightarrow \cdots
$$

where each $F_{i}$ and $F^{i}$ is flat, such that $M \cong \operatorname{Im}\left(F_{0} \rightarrow C \otimes_{R} F^{0}\right)$ and the sequence $\operatorname{Hom}_{R}(C, I) \otimes_{R} \mathbb{Z}$ is exact for each injective $R$-module $I$. The exact sequence $\mathbb{Z}$ is called a complete $F_{C}$-resolution of $T$. 
Note that, when $C=R$, these definitions above correspond to the definitions of Gorenstein projective, injective and flat modules and complete projective, injective and flat resolutions.

We will denote the classes of all $G_{C}$-proj, $G_{C}$-inj and $G_{C}$-flat $R$-modules by $\mathcal{G} \mathcal{P}_{C}(R), \mathcal{G I}_{C}(R)$ and $\mathcal{G F}_{C}(R)$, respectively.

Remark 2.7. (1) From [15] we know that every $C$-projective $R$-module is $G_{C^{-}}$ proj and the class $\mathcal{G P} C(R)$ is projectively resolving and closed under arbitrary direct sums and summands.

(2) Similar to the proofs in [15] we can easily get that every $C$-injective $R$-module is $G_{C}$-inj, the class $\mathcal{G I}_{C}(R)$ is injectively resolving and closed under arbitrary direct products and summands, every $C$-flat $R$-module is $G_{C}$-flat, and the class $\mathcal{G F}_{C}(R)$ is closed under arbitrary direct sums and summands.

Definition 2.8 ([9, Definition 4.1]). The Auslander class $\mathcal{A}_{C}(R)$ with respect to $C$ consists of all $R$-modules $M$ satisfying

(1) $\operatorname{Tor}_{\geqslant 1}^{R}(C, M)=0=\operatorname{Ext}_{R}^{\geqslant 1}\left(C, C \otimes_{R} M\right)$ and

(2) The natural evaluation map $\mu_{M}: M \rightarrow \operatorname{Hom}_{R}\left(C, C \otimes_{R} M\right)$ is an isomorphism.

Dually, the Bass class $\mathcal{B}_{C}(R)$ with respect to $C$ consists of all $R$-modules $N$ satisfying

(1) $\operatorname{Ext}_{R}^{\geqslant 1}(C, N)=0=\operatorname{Tor}_{\geqslant 1}^{R}\left(C, \operatorname{Hom}_{R}(C, N)\right)$ and

(2) The natural evaluation map $\nu_{N}: C \otimes_{R} \operatorname{Hom}_{R}(C, N) \rightarrow N$ is an isomorphism.

\section{Some basic propositions and lemmas}

In the following part of this paper, we distinguish $S$-modules from $R$-modules by marking the former with a tilde, e.g., $\tilde{N}$.

Proposition 3.1. Let $\varphi: R \rightarrow S$ be a homomorphism of rings and $\tilde{C}$ a semidualizing $S$-module. Then the following hold:

(1) If $\tilde{F}_{\tilde{C}}$ is a $\tilde{C}$-flat $S$-module and $F$ is a flat $R$-module, then $\tilde{F}_{\tilde{C}} \otimes_{R} F$ is a $\tilde{C}$-flat $S$-module.

(2) If $\tilde{P}_{\tilde{C}}$ is a $\tilde{C}$-projective $S$-module and $P$ is a projective $R$-module, then $\tilde{P}_{\tilde{C}} \otimes_{R} P$ is a $\tilde{C}$-projective $S$-module.

(3) If $\tilde{F}_{\tilde{C}}$ is a $\tilde{C}$-flat $S$-module and $I$ is an injective $R$-module, then $\operatorname{Hom}_{R}\left(\tilde{F}_{\tilde{C}}, I\right)$ is a $\tilde{C}$-injective $S$-module.

(4) If $\tilde{I}_{\tilde{C}}$ is a $\tilde{C}$-injective $S$-module and $F$ is a flat $R$-module, then $\operatorname{Hom}_{R}\left(F, \tilde{I}_{\tilde{C}}\right)$ is a $\tilde{C}$-injective $S$-module.

If $S$ is coherent, the following hold:

(5) If $\tilde{I}_{\tilde{C}}$ is a $\tilde{C}$-injective $S$-module and $F$ is a flat $R$-module, then $\tilde{I}_{\tilde{C}} \otimes_{R} F$ is a $\tilde{C}$-injective $S$-module.

(6) If $\tilde{F}_{\tilde{C}}$ is a $\tilde{C}$-flat $S$-module and $P$ is a projective $R$-module, then $\operatorname{Hom}_{R}\left(P, \tilde{F}_{\tilde{C}}\right)$ is a $\tilde{C}$-flat $S$-module. 
(7) If $\tilde{I}_{\tilde{C}}$ is a $\tilde{C}$-injective $S$-module and $I$ is an injective $R$-module, then $\operatorname{Hom}_{R}\left(\tilde{I}_{\tilde{C}}, I\right)$ is a $\tilde{C}$-flat $S$-module.

Proof. All seven results are straightforward to verify. As an example, consider (6): there is an isomorphism $\operatorname{Hom}_{R}\left(P, \tilde{C} \otimes_{S} \tilde{F}\right) \cong \operatorname{Hom}_{R}(P, \tilde{F}) \otimes_{S} \tilde{C}$ under the condition that $P$ is a projective $R$-module since $\tilde{C}$ admits a degreewise finite projective $S$-module resolution. We can also get that $\operatorname{Hom}_{R}(P, \tilde{F})$ is a flat $S$-module since the class of flat $S$-modules is closed under arbitrary direct

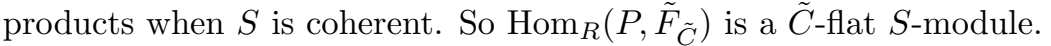

Also note that the fourth follows directly from the following isomorphism and the fact that $\operatorname{Hom}_{R}(F, \tilde{I})$ is an injective $S$-module.

$$
\operatorname{Hom}_{R}\left(F, \operatorname{Hom}_{S}(\tilde{C}, \tilde{I})\right) \cong \operatorname{Hom}_{S}\left(\tilde{C}, \operatorname{Hom}_{R}(F, \tilde{I})\right) .
$$

The following lemma is a generalization of [9, Proposition 3.2].

Lemma 3.2. Let $\varphi: R \rightarrow S$ be a homomorphism of rings with $f d_{R}(S)<\infty$ and $C$ a semidualizing $R$-module. Then $C \otimes_{R} S$ is a semidualizing $S$-module.

Proof. We first show that $C \otimes_{R} S$ admits a degreewise finite projective $S$ module resolution. Let $\mathbb{P}$ be a degreewise finite projective $R$-module resolution of $C$. Since $\operatorname{fd}_{R}(S)<\infty, S \in \mathcal{A}_{C}(R)$ by [9, Proposition 3.1] and [9, Corollary 6.2]. Thus, $\operatorname{Tor}_{\geqslant 1}^{R}(C, S)=0$. It follows that the sequence $\mathbb{P} \otimes_{R} S$ is exact and it is trivial that $\mathbb{P} \otimes_{R} S$ is the degreewise finite projective $S$-module resolution of $C \otimes_{R} S$.

Secondly, we show that the natural homothety map

$$
S \rightarrow \operatorname{Hom}_{S}\left(C \otimes_{R} S, C \otimes_{R} S\right)
$$

is an isomorphism. Similar to the proof of [9, Proposition 3.2], we only need to show that $\operatorname{Hom}_{R}(C, C) \otimes_{R} S \cong \operatorname{Hom}_{R}\left(C, C \otimes_{R} S\right)$. Since $\operatorname{fd}_{R}(S)=n<\infty$ for some non-negative integer $n$, we have the following exact sequence of $R$ modules:

$$
\mathbb{L}=0 \longrightarrow F_{n} \longrightarrow \cdots \longrightarrow F_{0} \longrightarrow S \longrightarrow 0
$$

where each $F_{i}$ is flat. Since $S \in \mathcal{A}_{C}(R)$, the sequence

$$
C \otimes_{R} \mathbb{L}=0 \longrightarrow C \otimes_{R} F_{n} \longrightarrow \cdots \longrightarrow C \otimes_{R} F_{0} \longrightarrow C \otimes_{R} S \longrightarrow 0
$$

is exact. By [9, Theorem 1], [9, Proposition 3.1] and [9, Corollary 6.3], we know that each kernel of $C \otimes_{R} \mathbb{L}$ belongs to $\mathcal{B}_{C}(R)$. So we have the following commutative diagram with exact rows:

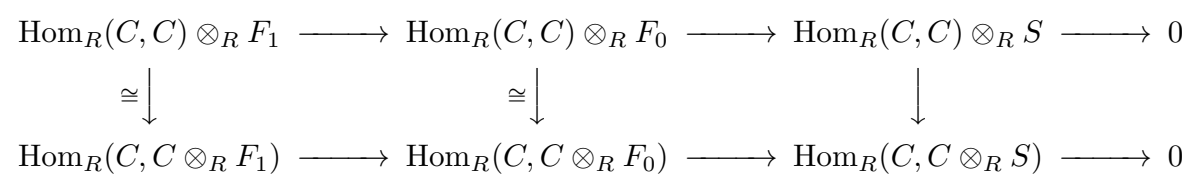

By the five lemma we can get our desired goal. 
Finally, we show that $\operatorname{Ext}_{S}^{i}\left(C \otimes_{R} S, C \otimes_{R} S\right)=0$. In fact, for $i \geqslant 1$, the first and third isomorphisms below are by definition while the second is Hom-tensor adjointness.

$$
\begin{aligned}
\operatorname{Ext}_{S}^{i}\left(C \otimes_{R} S, C \otimes_{R} S\right) & \cong \mathrm{H}_{-i} \operatorname{Hom}_{S}\left(\mathbb{P} \otimes_{R} S, C \otimes_{R} S\right) \\
& \cong \mathrm{H}_{-i} \operatorname{Hom}_{R}\left(\mathbb{P}, C \otimes_{R} S\right) \\
& \cong \operatorname{Ext}_{R}^{i}\left(C, C \otimes_{R} S\right) .
\end{aligned}
$$

On the other hand, from the exact sequence $C \otimes_{R} \mathbb{L}$ we know that $\operatorname{Ext}_{R}^{i}\left(C, C \otimes_{R}\right.$ $S) \cong \operatorname{Ext}_{R}^{n+i}\left(C, C \otimes_{R} F_{n}\right) \cong \operatorname{Ext}_{R}^{n+i}(C, C) \otimes_{R} F_{n}=0$ for any $i \geqslant 1$ by $[9$, Lemma 1.1].

From these three steps above, we get that $C \otimes_{R} S$ is a semidualizing $S$ module.

Proposition 3.3. Let $\varphi: R \rightarrow S$ be a homomorphism of rings with $f d_{R}(S)<$ $\infty, C$ a semidualizing $R$-module and $\tilde{C}=C \otimes_{R} S$. Then the following hold:

(1) If $F_{C}$ is a $C$-flat $R$-module and $\tilde{F}$ is a flat $S$-module, then $F_{C} \otimes_{R} \tilde{F}$ is a $\tilde{C}$-flat $S$-module.

(2) If $P_{C}$ is a $C$-projective $R$-module and $\tilde{P}$ is a projective $S$-module, then $P_{C} \otimes_{R} \tilde{P}$ is a $\tilde{C}$-projective $S$-module.

(3) If $F_{C}$ is a $C$-flat $R$-module and $\tilde{I}$ is an injective $S$-module, then $\operatorname{Hom}_{R}\left(F_{C}, \tilde{I}\right)$ is a $\tilde{C}$-injective $S$-module.

(4) If $I_{C}$ is a $C$-injective $R$-module and $\tilde{F}$ is a flat $S$-module, then $\operatorname{Hom}_{R}\left(\tilde{F}, I_{C}\right)$ is a $\tilde{C}$-injective $S$-module.

Proof. We only prove (3). Let $F$ be a flat $R$-module and $\tilde{I}$ an injective $S$ module. The result is directly obtained from the following isomorphisms and Proposition 3.1(4).

$\operatorname{Hom}_{R}\left(C \otimes_{R} F, \tilde{I}\right) \cong \operatorname{Hom}_{S}\left(C \otimes_{R} F \otimes_{R} S, \tilde{I}\right) \cong \operatorname{Hom}_{R}\left(F, \operatorname{Hom}_{S}\left(C \otimes_{R} S, \tilde{I}\right)\right)$.

Lemma 3.4. Let $C$ be a semidualizing $R$-module. Then the following hold:

(1) If $\mathbb{X}$ is a complete $P_{C}$-resolution, then the sequence $\mathbb{X} \otimes_{R} M$ is exact for any $R$-module $M \in F(R)$.

(2) If $\mathbb{X}$ is a complete $P_{C}$-resolution, then the sequence $\operatorname{Hom}_{R}\left(\mathbb{X}, C \otimes_{R} M\right)$ is exact for any $R$-module $M \in P(R)$.

(3) If $\mathbb{Y}$ is a complete $I_{C}$-resolution, then the sequence $\operatorname{Hom}_{R}(M, \mathbb{Y})$ is exact for any $R$-module $M \in P(R)$.

(4) If $\mathbb{Y}$ is a complete $I_{C}$-resolution, then the sequence $\operatorname{Hom}_{R}\left(\operatorname{Hom}_{R}(C, M)\right.$, $\mathbb{Y})$ is exact for any $R$-module $M \in I(R)$.

(5) If $\mathbb{Z}$ is a complete $F_{C}$-resolution, then the sequence $\mathbb{Z} \otimes_{R} M$ is exact for any $R$-module $M \in F(R)$.

(6) If $\mathbb{Z}$ is a complete $F_{C}$-resolution, then the sequence $\mathbb{Z} \otimes_{R} \operatorname{Hom}_{R}(C, M)$ is exact for any $R$-module $M \in I(R)$. 
Proof. The proofs of all six results are similar, so we only give the proof of (4).

Let $J$ be an injective $R$-module. Consider a complete $I_{C}$-resolution $\mathbb{Y}$ and an $R$-module $M$ with $\operatorname{id}_{R}(M)=n<\infty$ for some non-negative integer $n$. Then we have an exact sequence of $R$-modules

$$
0 \longrightarrow M \longrightarrow I_{0} \longrightarrow \cdots \longrightarrow I_{n} \longrightarrow 0,
$$

where each $I_{i}$ is injective. Let $K_{i}=\operatorname{Ker}\left(I_{i} \rightarrow I_{i+1}\right)$ for $1 \leqslant i \leqslant n-1$ and $K_{0}=M$. Consider the following short exact sequence:

$$
\mathbb{L}=0 \longrightarrow K_{n-1} \longrightarrow I_{n-1} \longrightarrow I_{n} \longrightarrow 0 .
$$

By [9, Proposition 3.1] and [9, Corollary 6.2], we know that $K_{n-1} \in \mathcal{B}_{C}(R)$. Thus, the sequence $\operatorname{Hom}_{R}(C, \mathbb{L})$ is exact. Also, we have $\operatorname{Tor}_{\geqslant 1}^{R}\left(C, \operatorname{Hom}_{R}\left(C, I_{n}\right)\right)$ $=0$ by $[9$, Theorem 1]. Then we get the following exact sequence of $R$-modules: $0 \longrightarrow \operatorname{Hom}_{R}\left(C, K_{n-1}\right) \otimes_{R} C \longrightarrow \operatorname{Hom}_{R}\left(C, I_{n-1}\right) \otimes_{R} C \longrightarrow \operatorname{Hom}_{R}\left(C, I_{n}\right) \otimes_{R} C \longrightarrow 0$.

So the sequence $\operatorname{Hom}_{R}\left(\operatorname{Hom}_{R}(C, \mathbb{L}), \operatorname{Hom}_{R}(C, J)\right)$ is exact by Hom-tensor adjointness. On the other hand, it is trivial that the sequence $\operatorname{Hom}_{R}\left(\operatorname{Hom}_{R}(C, \mathbb{L})\right.$, $J)$ is exact.

Now consider the short exact sequence of $R$-complexes:

$$
\begin{aligned}
0 \longrightarrow \operatorname{Hom}_{R}\left(\operatorname{Hom}_{R}\left(C, I_{n}\right), \mathbb{Y}\right) \longrightarrow \operatorname{Hom}_{R}\left(\operatorname{Hom}_{R}\left(C, I_{n-1}\right), \mathbb{Y}\right) \\
\quad \longrightarrow \operatorname{Hom}_{R}\left(\operatorname{Hom}_{R}\left(C, K_{n-1}\right), \mathbb{Y}\right) \longrightarrow 0 .
\end{aligned}
$$

Since $\operatorname{Hom}_{R}\left(\operatorname{Hom}_{R}\left(C, I_{n-1}\right), \mathbb{Y}\right)$ and $\operatorname{Hom}_{R}\left(\operatorname{Hom}_{R}\left(C, I_{n}\right), \mathbb{Y}\right)$ are exact, so is $\operatorname{Hom}_{R}\left(\operatorname{Hom}_{R}\left(C, K_{n-1}\right), \mathbb{Y}\right)$.

The argument above can be applied successively until we conclude that the sequence $\operatorname{Hom}_{R}\left(\operatorname{Hom}_{R}(C, M), \mathbb{Y}\right)$ is exact. This completes our proof.

Lemma 3.5 ([15, Lemma 1.7]). The class of $R$-modules admitting a degreewise finite projective (resp., free) resolution is closed under direct summands, extensions, kernels of epimorphisms and cokernels of monomorphisms.

Lemma 3.6. Consider the following exact sequence of $R$-modules:

$$
\mathbb{X}=\cdots \longrightarrow P_{1} \longrightarrow P_{0} \longrightarrow C \otimes_{R} P^{0} \longrightarrow C \otimes_{R} P^{1} \longrightarrow \cdots
$$

where each $P_{i}, P^{i}$ is finitely generated projective. Then the following are equivalent:

(1) $\operatorname{Hom}_{R}(\mathbb{X}, C)$ is exact.

(2) $\mathbb{X}$ is a complete $P_{C}$-resolution.

(3) $\mathbb{X}$ is a complete $F_{C}$-resolution.

Proof. Note that $C \otimes_{R} P$ admits a degreewise finite projective resolution for any finitely generated projective $R$-module $P$, so by the lemma above, $[9$, Lemma 1.1] and [9, Lemma 1.2], we can easily get our desired result.

Proposition 3.7. Let $R$ be a coherent ring. Suppose that every flat $R$-module has finite projective dimension. Then every $G_{C}$-proj $R$-module is $G_{C}$-flat. 
Proof. By [15, Proposition 2.12], similar to the proof of [7, Proposition 3.4], we only need to show that every $C$-flat $R$-module has finite $C$-projective dimension when every flat $R$-module has finite projective dimension. Let $F$ be a flat $R$ module and assume that $\operatorname{pd}_{R}(F)=n<\infty$ for some non-negative integer $n$. Consider the exact sequence of $R$-modules:

$$
0 \longrightarrow P_{n} \longrightarrow \cdots \longrightarrow P_{0} \longrightarrow F \longrightarrow 0
$$

where each $P_{i}$ is projective. Since $F \in \mathcal{A}_{C}$ by $[9$, Theorem 1$], \operatorname{Tor}_{\geqslant 1}^{R}(C, F)=0$. Thus, the sequence

$$
0 \longrightarrow C \otimes_{R} P_{n} \longrightarrow \cdots \longrightarrow C \otimes_{R} P_{0} \longrightarrow C \otimes_{R} F \longrightarrow 0
$$

is exact, so $\mathcal{P}_{C}-\operatorname{pd}_{R}\left(C \otimes_{R} F\right) \leqslant n<\infty$.

Proposition 3.8. Let $M$ be a $G_{C}$-proj $R$-module which admits a degreewise finite projective resolution. Then $M$ is $G_{C}$-flat.

Proof. Assume that $M$ is a $G_{C}$-proj $R$-module with a degreewise finite projective resolution. We now build a short exact sequence of $R$-modules

$$
0 \longrightarrow M \longrightarrow C \otimes_{R} H^{0} \longrightarrow M^{\prime} \longrightarrow 0,
$$

where $H^{0}$ is finitely generated projective and $M^{\prime}$ has the same properties as $M$.

Since $M$ is a $G_{C}$-proj $R$-module, we have a short exact sequence of $R$ modules

$$
0 \rightarrow M \rightarrow C \otimes_{R} P^{0} \rightarrow K \rightarrow 0,
$$

where $P^{0}$ is projective and $K$ is $G_{C}$-proj. Since $P^{0}$ is projective, we can choose another projective $R$-module $Q^{0}$ such that $P^{0} \oplus Q^{0}=F^{0}$, where $F^{0}$ is a free $R$ module. Adding $0 \rightarrow 0 \rightarrow C \otimes_{R} Q^{0} \rightarrow C \otimes_{R} Q^{0} \rightarrow 0$ to (*) we get a short exact sequence of $R$-modules $0 \rightarrow M \rightarrow C \otimes_{R} F^{0} \rightarrow N \rightarrow 0$ with $N=C \otimes_{R} Q^{0} \oplus K$ also $G_{C}$-proj.

Since $C \otimes_{R} F^{0}$ is a direct sum of copies of $C$, the image of the finitely generated $R$-module $M$ is contained in a finite direct sum of copies of $C$. That is, the image of $M$ is contained in a finitely generated submodule $C \otimes_{R} H^{0}$ of $C \otimes_{R} F^{0}$. Thus, we have the following commutative diagram with exact rows:

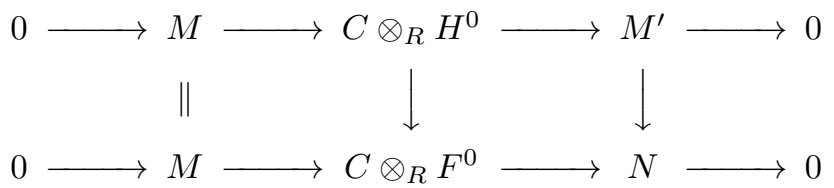

Let $P$ be a projective $R$-module and $\mathcal{F}=\operatorname{Hom}_{R}\left(-, C \otimes_{R} P\right)$. Since $C \otimes_{R} H^{0}$ and $N$ are $G_{C}$-proj, we have $\operatorname{Ext}_{R}^{1}\left(N, C \otimes_{R} P\right)=0=\operatorname{Ext}_{R}^{1}\left(C \otimes_{R} H^{0}, C \otimes_{R} P\right)$ by $[15$, Proposition 2.2]. Hence, applying $\mathcal{F}$ to the above commutative diagram yields another commutative diagram: 
TRANSFER PROPERTIES OF GORENSTEIN HOMOLOGICAL DIMENSION 1205

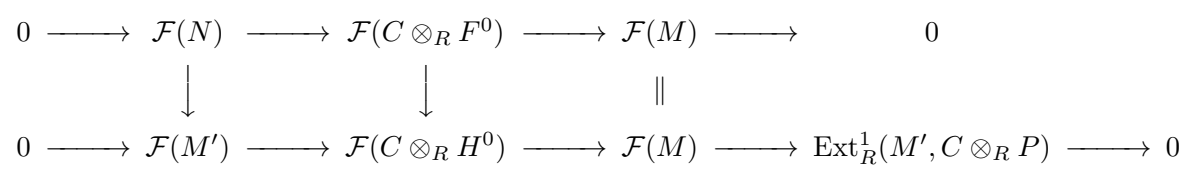

A routine diagram chase shows that $\operatorname{Ext}_{R}^{1}\left(M^{\prime}, C \otimes_{R} P\right)=0$. By [15, Proposition 2.12 ] and Lemma 3.5 , we know that $M^{\prime}$ is a $G_{C}$-proj $R$-module and admits a degreewise finite projective resolution.

Continue the above procedure we get an exact sequence of $R$-modules

$$
\mathbb{X}=\cdots \longrightarrow P_{1} \longrightarrow P_{0} \longrightarrow C \otimes_{R} H^{0} \longrightarrow C \otimes_{R} H^{1} \longrightarrow \cdots,
$$

where each $P_{i}$ and $H^{i}$ is finitely generated projective, such that the sequence $\operatorname{Hom}_{R}\left(\mathbb{X}, C \otimes_{R} P\right)$ is exact. So by Lemma 3.6 we get our desired result.

Corollary 3.9. Let $M$ be an $R$-module which admits a degreewise finite projective resolution and $n$ a non-negative integer. If $G_{C}-p d_{R}(M)<\infty$, then the following are equivalent:

(1) $G_{C}-p d_{R}(M) \leqslant n$.

(2) $\operatorname{Ext}_{R}^{>n}(M, F)=0$ for all $R$-module $F$ with $\mathcal{F}_{C}-\operatorname{pd}_{R}(F)<\infty$.

(3) $\operatorname{Ext}_{R}^{>n}(M, F)=0$ for all $C$-flat $R$-module $F$.

(4) $\operatorname{Ext}_{R}^{>n}(M, C)=0$.

Proof. By [9, Lemma 1.2], the proof is similar to [2, Theorem 1.2.7].

\section{Transfer properties}

One should compare the results in this section with Proposition 3.1 and Proposition 3.3.

Proposition 4.1. Let $\varphi: R \rightarrow S$ be a homomorphism of rings and $\tilde{C}$ a semidualizing $S$-module. Then the following hold:

(1) $\tilde{B}$ is a $G_{\tilde{C}}$-flat $S$-module if and only if $\tilde{B} \otimes_{R} F$ is a $G_{\tilde{C}}$-flat $S$-module for any flat $R$-module $F$.

(2) $\tilde{A}$ is a $G_{\tilde{C}}$-proj $S$-module if and only if $\tilde{A} \otimes_{R} P$ is a $G_{\tilde{C}}$-proj $S$-module for any projective $R$-module $P$.

Proof. By Proposition 3.1(1) and (2) and the definitions of $G_{\tilde{C}^{-}}$flat and $G_{\tilde{C}^{-}}$ proj modules respectively, the proof of (1) and (2) is easy.

Proposition 4.2. Let $\varphi: R \rightarrow S$ be a homomorphism of rings and $\tilde{C}$ a semidualizing $S$-module. If $S$ is coherent, then the following are equivalent:

(1) $\tilde{B}$ is a $G_{\tilde{C}}$-flat $S$-module.

(2) $\operatorname{Hom}_{R}(\tilde{B}, E)$ is a $G_{\tilde{C}}$-inj $S$-module for any injective $R$-module $E$.

(3) $\operatorname{Hom}_{R}(\tilde{B}, E)$ is a $G_{\tilde{C}}$-inj $S$-module for any faithfully injective $R$-module E.

(4) $\tilde{B}$ admits a co-proper right $\mathcal{F}_{\tilde{C}}(S)$-resolution and $\operatorname{Tor}_{\geqslant 1}^{S}\left(\operatorname{Hom}_{S}(\tilde{C}, \tilde{J}), \tilde{B}\right)$ $=0$ for any injective $S$-module $\tilde{J}$. 
Proof. $(1) \Rightarrow(2)$ It follows directly from Proposition 3.1(3) and standard Homtensor adjointness.

$(2) \Rightarrow(3)$ It is trivial.

$(3) \Rightarrow(4)$ Since the class $F_{\tilde{C}}(S)$ is preenveloping when $S$ is coherent by $[9$, Proposition 5.3], the proof is similar to [2, Theorem 6.4.2].

$(4) \Rightarrow(1)$ From the definition of $G_{\tilde{C}}$-flat modules and Proposition 3.1(7) we can easily get our desired result.

Similar to the proof of [7, Theorem 3.7] we get the following result:

Theorem 4.3. Let $S$ be a coherent ring and $\tilde{C}$ a semidualizing $S$-module. If $\tilde{M}_{0} \rightarrow \tilde{M}_{1} \rightarrow \tilde{M}_{2} \rightarrow \cdots$ is a sequence of $G_{\tilde{C}}$-flat $S$-modules, then the direct limit $\lim _{n} M_{n}$ is again $G_{\tilde{C}^{-}}$flat.

Theorem 4.4. Let $\varphi: R \rightarrow S$ be a homomorphism of rings and $\tilde{C}$ a semidualizing $S$-module. If $S$ is coherent, then $\tilde{H}$ is a $G_{\tilde{C}}$-inj $S$-module if and only if $\operatorname{Hom}_{R}(F, \tilde{H})$ is a $G_{\tilde{C}}$-inj $S$-module for any flat $R$-module $F$ holds under each of the next three conditions.

(1) $F(S)=P(S)$.

(2) $F(R)=P(R)$.

(3) $\mathcal{P}_{\tilde{C}}-p d_{S}\left(\tilde{C} \otimes_{S} \tilde{F}\right)<\infty$ for any flat $S$-module $\tilde{F}$.

Proof. $(\Rightarrow)$ Let $\tilde{\mathbb{Y}}$ be a complete $I_{\tilde{C}}$-resolution of a $G_{\tilde{C}}$-inj $S$-module $\tilde{H}$ and $F$ a flat $R$-module.

Firstly, we have that, under either assumption, $F \otimes_{R} S$ has finite projective dimension over $S$. In fact, for (3), assume that $\mathcal{P}_{\tilde{C}}-\operatorname{pd}_{S}\left(\tilde{C} \otimes_{S} F \otimes_{R} S\right)=$ $t<\infty$ for some non-negative integer $t$, so there exists a left $\mathcal{P}_{\tilde{C}}$-resolution of $\tilde{C} \otimes_{S} F \otimes_{R} S$

$$
\tilde{\mathbb{X}}=0 \longrightarrow \tilde{C} \otimes_{S} \tilde{P}_{t} \longrightarrow \cdots \longrightarrow \tilde{C} \otimes_{S} \tilde{P}_{0} \longrightarrow \tilde{C} \otimes_{S} F \otimes_{R} S \longrightarrow 0,
$$

where each $\tilde{P}_{i}$ is projective. Since each $\tilde{P}_{i}$ and $F \otimes_{R} S$ belong to $\mathcal{A}_{\tilde{C}}(S)$ by $[9$, Theorem 1], the sequence $\operatorname{Hom}_{S}(\tilde{C}, \tilde{\mathbb{X}})$ is exact and each $\mu_{\tilde{P}_{i}}$ and $\mu_{F \otimes_{R} S}$ is an isomorphism by $\left[9\right.$, Lemma 4.1]. Thus, $\operatorname{Hom}_{S}(\tilde{C}, \tilde{\mathbb{X}})$ is isomorphic to an exact sequence of the form:

$$
0 \longrightarrow \tilde{P}_{t} \longrightarrow \cdots \longrightarrow \tilde{P}_{0} \longrightarrow F \otimes_{R} S \longrightarrow 0 .
$$

It follows that $F \otimes_{R} S$ has finite projective dimension over $S$. For (1) and (2), it is trivial.

Secondly, by the isomorphism $\operatorname{Hom}_{R}(F, \tilde{\mathbb{Y}}) \cong \operatorname{Hom}_{S}\left(F \otimes_{R} S, \tilde{\mathbb{Y}}\right)$ and Lemma $3.4(3)$, we know that the sequence $\operatorname{Hom}_{R}(F, \tilde{Y})$ is exact.

Thirdly, by Proposition 3.1(4) we only need to prove that, for any injective $S$-module $\tilde{J}, \operatorname{Hom}_{S}\left(\operatorname{Hom}_{S}(\tilde{C}, \tilde{J}), \operatorname{Hom}_{R}(F, \tilde{\mathbb{Y}})\right)$ is exact. In fact,

$$
\begin{aligned}
\operatorname{Hom}_{S}\left(\operatorname{Hom}_{S}(\tilde{C}, \tilde{J}), \operatorname{Hom}_{R}(F, \tilde{Y})\right) & \cong \operatorname{Hom}_{S}\left(\operatorname{Hom}_{S}(\tilde{C}, \tilde{J}), \operatorname{Hom}_{S}\left(F \otimes_{R} S, \tilde{\mathbb{Y}}\right)\right) \\
& \cong \operatorname{Hom}_{S}\left(\operatorname{Hom}_{S}(\tilde{C}, \tilde{J}) \otimes_{R} F, \tilde{\mathbb{Y}}\right)
\end{aligned}
$$


is exact by Proposition 3.1(5).

From these three steps above, we know that $\operatorname{Hom}_{R}(F, \tilde{H})$ is a $G_{\tilde{C}^{-}}$-inj $S$ module.

$(\Leftarrow)$ It is trivial.

Remark 4.5. It is easy to verify that when $F$, in the above theorem, is a projective $R$-module, then the equivalence holds without any additional conditions.

The next lemma follows readily from [9, Proposition 3.1], [9, Proposition $5.2]$ and [9, Corollary 6.4].

Lemma 4.6. Let $\tilde{C}$ be a semidualizing $S$-module. Consider the following short exact sequence of $S$-modules:

$$
0 \longrightarrow \tilde{M} \longrightarrow \tilde{N} \longrightarrow \tilde{K} \longrightarrow 0
$$

When $\tilde{M}$ is $\tilde{C}$-injective, then $\tilde{K}$ is $\tilde{C}$-injective if and only if $\tilde{N}$ is $\tilde{C}$-injective. If $\tilde{M}$ and $\tilde{K}$ are $\tilde{C}$-injective, then the above short exact sequence is split.

Theorem 4.7. Let $\varphi: R \rightarrow S$ be a homomorphism of rings and $\tilde{C}$ a semidualizing $S$-module. If $S$ is Artinian, then the following are equivalent:

(1) $\tilde{H}$ is a $G_{\tilde{C}}$-inj $S$-module.

(2) $\operatorname{Hom}_{R}(\tilde{H}, E)$ is a $G_{\tilde{C}}$-flat $S$-module for any injective $R$-module $E$. E.

(3) $\operatorname{Hom}_{R}(\tilde{H}, E)$ is a $G_{\tilde{C}}$-flat $S$-module for any faithfully injective $R$-module

(4) $\tilde{H} \otimes_{R} F$ is a $G_{\tilde{C}}$-inj $S$-module for any flat $R$-module $F$.

(5) $\tilde{H} \otimes_{R} F$ is a $G_{\tilde{C}}$-inj $S$-module for any faithfully flat $R$-module $F$.

Proof. $(1) \Rightarrow(2)$ Let $\tilde{H}$ be a $G_{\tilde{C}}$-inj $S$-module, $E$ an injective $R$-module and the exact sequence of $S$-modules

$$
\tilde{\mathbb{I}}=\cdots \longrightarrow \operatorname{Hom}_{S}\left(\tilde{C}, \tilde{I}^{1}\right) \longrightarrow \operatorname{Hom}_{S}\left(\tilde{C}, \tilde{I}^{0}\right) \longrightarrow \tilde{I}_{0} \longrightarrow \tilde{I}_{1} \longrightarrow \cdots
$$

a complete $I_{\tilde{C}}$-resolution of $\tilde{H}$. By Proposition $3.1(7)$ we only need to prove that, for any injective $S$-module $\tilde{I}, \operatorname{Tor}_{1}^{S}\left(\operatorname{Hom}_{S}(\tilde{C}, \tilde{I}), \operatorname{Hom}_{R}\left(\tilde{K}^{t}, E\right)\right)=0$ and $\operatorname{Tor}_{1}^{S}\left(\operatorname{Hom}_{S}(\tilde{C}, \tilde{I}), \operatorname{Hom}_{R}\left(\tilde{K}_{j}, E\right)\right)=0$, where $\tilde{K}^{t}$ and $\tilde{K}_{j}$ are cokernels of $\tilde{\mathbb{I}}$ for all $t \geqslant 0, j \geqslant 0$. Since $S$ is Artinian, we have that $\tilde{I}=\bigoplus_{\Lambda} \tilde{E}_{\alpha}$ for some index set $\Lambda$, where $\tilde{E}_{\alpha}$ is an injective envelope of some simple $S$-module for any $\alpha \in \Lambda$. Then $\operatorname{Tor}_{1}^{S}\left(\operatorname{Hom}_{S}(\tilde{C}, \tilde{I}), \operatorname{Hom}_{R}\left(\tilde{K}^{t}, E\right)\right) \cong \bigoplus_{\Lambda} \operatorname{Tor}_{1}^{S}\left(\operatorname{Hom}_{S}\left(\tilde{C}, \tilde{E}_{\alpha}\right), \operatorname{Hom}_{R}\left(\tilde{K}^{t}\right.\right.$, $E)) \cong \bigoplus_{\Lambda} \operatorname{Hom}_{R}\left(\operatorname{Ext}_{R}^{1}\left(\operatorname{Hom}_{S}\left(\tilde{C}, \tilde{E}_{\alpha}\right), \tilde{K}^{t}\right), E\right)=0$ by [4, p. 16, Exercise 2] and $\left[4\right.$, Theorem 3.2.13] for all $t \geqslant 0$. Similarly for each $\tilde{K}_{j}$. Thus, $\operatorname{Hom}_{R}(\tilde{H}, E)$ is a $G_{\tilde{C}}$-flat $S$-module.

$(2) \Rightarrow(3)$ It is trivial.

$(3) \Rightarrow(1)$ Let $E$ be a faithfully injective $R$-module and $-^{+}=\operatorname{Hom}_{R}(-, E)$. Since $\tilde{H}^{+}$is a $G_{\tilde{C}}$-flat $S$-module, there exists a complete $F_{\tilde{C}}$-resolution

$$
\tilde{\mathbb{Z}}=\cdots \longrightarrow \tilde{F}_{1} \longrightarrow \tilde{F}_{0} \longrightarrow \tilde{C} \otimes_{S} \tilde{F}^{0} \longrightarrow \tilde{C} \otimes_{S} \tilde{F}^{1} \longrightarrow \cdots,
$$


where each $\tilde{F}_{i}$ and $\tilde{F}^{i}$ is flat, such that $\tilde{\mathbb{Z}}_{l}=\cdots \rightarrow \tilde{F}_{1} \rightarrow \tilde{F}_{0} \rightarrow \tilde{H}^{+} \rightarrow 0$ and $\tilde{\mathbb{Z}}_{r}=0 \rightarrow \tilde{H}^{+} \rightarrow \tilde{C} \otimes_{S} \tilde{F}^{0} \rightarrow \tilde{C} \otimes_{S} \tilde{F}^{1} \rightarrow \cdots$ is exact. So we have exact sequences of $S$-modules

$$
\begin{gathered}
\tilde{\mathbb{Z}}_{l}^{+}=0 \longrightarrow \tilde{H}^{++} \longrightarrow \tilde{F}_{0}^{+} \longrightarrow \tilde{F}_{1}^{+} \longrightarrow \cdots \\
\tilde{\mathbb{Z}}_{r}^{+}=\cdots \longrightarrow\left(\tilde{C} \otimes_{S} \tilde{F}^{1}\right)^{+} \longrightarrow\left(\tilde{C} \otimes_{S} \tilde{F}^{0}\right)^{+} \longrightarrow \tilde{H}^{++} \longrightarrow 0 .
\end{gathered}
$$

By Lemma 4.6 , we can successively take injective $S$-modules $\tilde{E}_{0}, \tilde{E}_{1}, \ldots$ and $\tilde{C}$-injective $S$-modules $\tilde{I}^{0}, \tilde{I}^{1}, \ldots$ such that

$$
\tilde{F}_{0}^{+} \oplus \tilde{E}_{0} \cong \tilde{F}_{0}^{+++}, \tilde{F}_{i}^{+} \oplus \tilde{E}_{i-1} \oplus \tilde{E}_{i} \cong\left(\tilde{F}_{i}^{+} \oplus \tilde{E}_{i-1}\right)^{++},
$$

$\left(\tilde{C} \otimes_{S} \tilde{F}^{0}\right)^{+} \oplus \tilde{I}^{0} \cong\left(\tilde{C} \otimes_{S} \tilde{F}^{0}\right)^{+++},\left(\tilde{C} \otimes_{S} \tilde{F}^{i}\right)^{+} \oplus \tilde{I}^{i-1} \oplus \tilde{I}^{i} \cong\left(\left(\tilde{C} \otimes_{S} \tilde{F}^{i}\right)^{+} \oplus \tilde{I}^{i-1}\right)^{++}$ for all $i=1,2, \ldots$ Adding the exact sequence $0 \rightarrow \tilde{E}_{i} \rightarrow \tilde{E}_{i} \rightarrow 0$ to $i+1$ positions and $i+2$ positions of the sequence $\tilde{\mathbb{Z}}_{l}^{+}$and the exact sequence $0 \rightarrow$ $\tilde{I}^{i} \rightarrow \tilde{I}^{i} \rightarrow 0$ to $i+2$ positions and $i+1$ positions of the sequence $\tilde{\mathbb{Z}}_{r}^{+}$for all $i=0,1, \ldots$, then we get an exact sequence of $S$-modules

$$
\cdots \longrightarrow\left(\left(\tilde{C} \otimes_{S} \tilde{F}^{1}\right)^{+} \oplus \tilde{I}^{0}\right)^{++} \longrightarrow\left(\tilde{C} \otimes_{S} \tilde{F}^{0}\right)^{+++} \longrightarrow \tilde{F}_{0}^{+++} \longrightarrow\left(\tilde{F}_{1}^{+} \oplus \tilde{E}_{0}\right)^{++} \longrightarrow \cdots \text {. }
$$

Thus, $\tilde{\mathbb{E}}=\cdots \rightarrow\left(\tilde{C} \otimes_{S} \tilde{F}^{1}\right)^{+} \oplus \tilde{I}^{0} \rightarrow\left(\tilde{C} \otimes_{S} \tilde{F}^{0}\right)^{+} \rightarrow \tilde{F}_{0}^{+} \rightarrow \tilde{F}_{1}^{+} \oplus \tilde{E}_{0} \rightarrow \cdots$ is exact and such that $\tilde{H} \cong \operatorname{Im}\left(\left(\tilde{C} \otimes_{S} \tilde{F}^{0}\right)^{+} \rightarrow \tilde{F}_{0}^{+}\right)$. We can also verify that $\tilde{F}_{0}^{+}$and each $\tilde{F}_{i}^{+} \oplus \tilde{E}_{i-1}$ are injective $S$-modules and $\left(\tilde{C} \otimes_{S} \tilde{F}^{0}\right)^{+}$and each $\left(\tilde{C} \otimes_{S} \tilde{F}^{i}\right)^{+} \oplus \tilde{I}^{i-1}$ are $\tilde{C}$-injective $S$-modules by Proposition 3.1(3). Now, let $\tilde{I}$ be an injective $S$-module. Since $S$ is Artinian, we have $\tilde{I} \cong \bigoplus_{\Lambda} \tilde{E}_{\alpha}$ for some index set $\Lambda$, where $\tilde{E}_{\alpha}$ is an injective envelope of some simple $S$-module for any $\alpha \in \Lambda$. On the one hand, $\operatorname{Hom}_{S}\left(\operatorname{Hom}_{S}\left(\tilde{C}, \tilde{E}_{\alpha}\right), \tilde{\mathbb{Z}}^{+}\right) \cong\left(\tilde{\mathbb{Z}} \otimes_{S} \operatorname{Hom}_{S}\left(\tilde{C}, \tilde{E}_{\alpha}\right)\right)^{+}$is exact. On the other hand, by [4, Theorem 3.2.11], we have

$$
\begin{aligned}
\operatorname{Hom}_{S}\left(\operatorname{Hom}_{S}\left(\tilde{C}, \tilde{E}_{\alpha}\right), \tilde{\mathbb{E}}^{++}\right) & \cong\left(\tilde{\mathbb{E}}^{+} \otimes_{S} \operatorname{Hom}_{S}\left(\tilde{C}, \tilde{E}_{\alpha}\right)\right)^{+} \\
& \cong\left(\operatorname{Hom}_{S}\left(\operatorname{Hom}_{S}\left(\tilde{C}, \tilde{E}_{\alpha}\right), \tilde{\mathbb{E}}\right)\right)^{++}
\end{aligned}
$$

is exact. So $\operatorname{Hom}_{S}\left(\operatorname{Hom}_{S}(\tilde{C}, \tilde{I}), \tilde{\mathbb{E}}\right) \cong \prod_{\Lambda} \operatorname{Hom}_{S}\left(\operatorname{Hom}_{S}\left(\tilde{C}, \tilde{E}_{\alpha}\right), \tilde{\mathbb{E}}\right)$ is exact. Hence, $\tilde{H}$ is $G_{\tilde{C}}$-inj.

$$
(1) \Rightarrow(4) \Rightarrow(5) \Rightarrow(1) \text { is easy. }
$$

Remark 4.8. It is easy to verify that when $F$, in the above theorem, is a finitely generated projective $R$-module, then the equivalence of (1) and (4) holds without the condition that $S$ is Artinian.

Lemma 4.9. Let $S$ be an Artinian ring and $\tilde{C}$ a semidualizing $S$-module. Then the class $\mathcal{G F}_{\tilde{C}}(S)$ is closed under arbitrary direct products.

Proof. Let $\tilde{M}=\prod_{i \in I} \tilde{M}_{i}$ and $\tilde{M}_{i} \in \mathcal{G F}_{\tilde{C}}(S)$ for all $i \in I$. There exists an exact sequence of $S$-modules $\cdots \rightarrow \tilde{F}_{1 i} \rightarrow \tilde{F}_{0 i} \rightarrow \tilde{C} \otimes_{S} \tilde{F}_{i}^{0} \rightarrow \tilde{C} \otimes_{S} \tilde{F}_{i}^{1} \rightarrow \cdots$ which is a complete $F_{\tilde{C}^{-}}$-resolution of $M_{i}$ for all $i \in I$ and let $\tilde{K}_{t i}$ and $\tilde{K}_{i}^{j}$ be cokernels of it for all $t \geqslant 0, j \geqslant 0$ and $i \in I$. Then $\cdots \rightarrow \prod_{i \in I} \tilde{F}_{1 i} \rightarrow$ $\prod_{i \in I} \tilde{F}_{0 i} \rightarrow \prod_{i \in I} \tilde{C} \otimes_{S} \tilde{F}_{i}^{0} \rightarrow \prod_{i \in I} \tilde{C} \otimes_{S} \tilde{F}_{i}^{1} \rightarrow \cdots$ is exact, $\prod_{i \in I} \tilde{F}_{k i}$ is flat 
and $\prod_{i \in I} \tilde{C} \otimes_{S} \tilde{F}_{i}^{h}$ is $\tilde{C}$-flat for all $k \geqslant 0, h \geqslant 0$. Let $\tilde{E}$ be an injective $S$-module. Since $S$ is Artinian, $\tilde{E}=\bigoplus_{\Lambda} \tilde{E}_{\alpha}$ for some index set $\Lambda$, where $\tilde{E}_{\alpha}$ is an injective envelope of some simple $S$-module for any $\alpha \in \Lambda$. Thus, $\operatorname{Tor}_{1}^{S}\left(\operatorname{Hom}_{S}(\tilde{C}, \tilde{E}), \prod_{i \in I} \tilde{K}_{t i}\right) \cong \bigoplus_{\Lambda} \prod_{i \in I} \operatorname{Tor}_{1}^{S}\left(\operatorname{Hom}_{S}\left(\tilde{C}, \tilde{E}_{\alpha}\right), \tilde{K}_{t i}\right)=0$ by [4, p. 16, Exercise 2] and [4, Theorem 3.2.26] for all $t \geqslant 0$. Similar for each $\prod_{i \in I} \tilde{K}_{i}^{j}$. Therefore $\tilde{M} \in \mathcal{G} \mathcal{F}_{\tilde{C}}(S)$.

Theorem 4.10. Let $\varphi: R \rightarrow S$ be a homomorphism of rings and $\tilde{C}$ a semidualizing $S$-module. If $S$ is Artinian, then $\tilde{B}$ is a $G_{\tilde{C}}$-flat $S$-module if and only if $\operatorname{Hom}_{R}(P, \tilde{B})$ is a $G_{\tilde{C}}$-flat $S$-module for any projective $R$-module $P$.

Proof. $(\Rightarrow)$ It follows directly from Lemma 4.9 and Remark 2.7.

$(\Leftarrow)$ It is trivial.

Remark 4.11. It is easy to verify that when $P$, in the above theorem, is a finitely generated projective $R$-module, then the equivalence holds without the condition that $S$ is Artinian.

In the following part of this section, let $C$ be a semidualizing $R$-module. We will denote $C \otimes_{R} S$ by $\tilde{C}$.

Proposition 4.12. Let $\varphi: R \rightarrow S$ be a homomorphism of rings with $f d_{R}(S)<$ $\infty$. Then the following hold:

(1) If $A$ is a $G_{C}$-flat $R$-module and $\tilde{F}$ is a flat $S$-module, then $A \otimes_{R} \tilde{F}$ is $a G_{\tilde{C}}$-flat $S$-module.

(2) If $A$ is a $G_{C}$-flat $R$-module and $\tilde{I}$ is an injective $S$-module, then $\operatorname{Hom}_{R}(A, \tilde{I})$ is a $G_{\tilde{C}}$-inj $S$-module.

(3) If $H$ is a $G_{C}$-proj $R$-module and $\tilde{P}$ is a projective $S$-module, then $H \otimes_{R} \tilde{P}$ is a $G_{\tilde{C}^{-}}$-proj $S$-module, provided $P(S) \subseteq P(R)$.

Proof. For (1) and (2), let $\mathbb{F}$ be a complete $F_{C}$-resolution of a $G_{C}$-flat $R$-module $A, \tilde{F}$ a flat $S$-module and $\tilde{I}$ an injective $S$-module.

(1) By Proposition 3.3(1), Lemma 3.4(5) and (6) and the following isomorphism,

$$
\operatorname{Hom}_{S}(\tilde{C}, \tilde{I}) \otimes_{S} \tilde{F} \otimes_{R} \mathbb{F} \cong \operatorname{Hom}_{R}(C, \tilde{I}) \otimes_{R} \mathbb{F} \otimes_{S} \tilde{F}
$$

we know that $A \otimes_{R} \tilde{F}$ is a $G_{\tilde{C}^{-}}$-flat $S$-module.

(2) On the one hand, $\operatorname{Hom}_{R}(\mathbb{F}, \tilde{I}) \cong \operatorname{Hom}_{S}\left(\mathbb{F} \otimes_{R} S, \tilde{I}\right)$ is exact by Lemma 3.4(5). On the other hand, by Proposition 3.3(3) we only need to prove that, for any injective $S$-module $\tilde{J}, \operatorname{Hom}_{S}\left(\operatorname{Hom}_{S}(\tilde{C}, \tilde{J}), \operatorname{Hom}_{R}(\mathbb{F}, \tilde{I})\right)$ is exact. In fact,

$$
\begin{aligned}
\operatorname{Hom}_{S}\left(\operatorname{Hom}_{S}(\tilde{C}, \tilde{J}), \operatorname{Hom}_{R}(\mathbb{F}, \tilde{I})\right) & \cong \operatorname{Hom}_{S}\left(\operatorname{Hom}_{R}(C, \tilde{J}), \operatorname{Hom}_{S}\left(\mathbb{F} \otimes_{R} S, \tilde{J}\right)\right) \\
& \cong \operatorname{Hom}_{S}\left(\operatorname{Hom}_{R}(C, \tilde{J}) \otimes_{R} \mathbb{F}, \tilde{I}\right)
\end{aligned}
$$

is exact by Lemma $3.4(6)$. Hence, $\operatorname{Hom}_{R}(A, \tilde{I})$ is a $G_{\tilde{C}^{-}}$-inj $S$-module. 
(3) Let $\mathbb{P}$ be a complete $P_{C}$-resolution of a $G_{C}$-proj $R$-module $H$ and $\tilde{P}$ a projective $S$-module. Suppose that $\tilde{Q}$ is a projective $S$-module, then by Proposition 3.3(2), Lemma 3.4(1) and (2) and the following isomorphism,

$$
\begin{aligned}
\operatorname{Hom}_{S}\left(\mathbb{P} \otimes_{R} \tilde{P}, \tilde{C} \otimes_{S} \tilde{Q}\right) & \cong \operatorname{Hom}_{S}\left(\mathbb{P} \otimes_{R} \tilde{P} \otimes_{S} S, \tilde{C} \otimes_{S} \tilde{Q}\right) \\
& \cong \operatorname{Hom}_{S}\left(\tilde{P}, \operatorname{Hom}_{R}\left(\mathbb{P}, C \otimes_{R} \tilde{Q}\right)\right)
\end{aligned}
$$

we know that $H \otimes_{R} \tilde{P}$ is a $G_{\tilde{C}^{-}}$-proj $S$-module.

Proposition 4.13. Let $\varphi: R \rightarrow S$ be a homomorphism of rings with $f d_{R}(S)<$ $\infty$. If $S$ is coherent, $H$ is a $G_{C}$-proj $R$-module and $\tilde{F}$ is a flat $S$-module, then $H \otimes_{R} \tilde{F}$ is a $G_{\tilde{C}}$-flat $S$-module, provided $F(S) \subseteq P(R)$.

Proof. Let $\mathbb{P}$ be a complete $P_{C}$-resolution of a $G_{C}$-proj $R$-module $H$ and $\tilde{F}$ a flat $S$-module. By Proposition 3.3(1) and Lemma 3.4(1) we only need to prove that, for any injective $S$-module $\tilde{I}, \operatorname{Hom}_{S}(\tilde{C}, \tilde{I}) \otimes_{S} \tilde{F} \otimes_{R} \mathbb{P}$ is exact. In fact, we first have the following isomorphism:

$\operatorname{Hom}_{\mathbb{Z}}\left(\operatorname{Hom}_{S}(\tilde{C}, \tilde{I}) \otimes_{S} \tilde{F} \otimes_{R} \mathbb{P}, \mathbb{Q} / \mathbb{Z}\right) \cong \operatorname{Hom}_{R}\left(\mathbb{P}, \operatorname{Hom}_{\mathbb{Z}}\left(\operatorname{Hom}_{S}(\tilde{C}, \tilde{I}) \otimes_{S} \tilde{F}, \mathbb{Q} / \mathbb{Z}\right)\right)$.

Secondly, since $\operatorname{Hom}_{\mathbb{Z}}\left(\operatorname{Hom}_{S}(\tilde{C}, \tilde{I}) \otimes_{S} \tilde{F}, \mathbb{Q} / \mathbb{Z}\right)$ is a $\tilde{C}$-flat $S$-module by Proposition 3.1(5) and (7). So $\operatorname{Hom}_{\mathbb{Z}}\left(\operatorname{Hom}_{S}(\tilde{C}, \tilde{I}) \otimes_{S} \tilde{F}, \mathbb{Q} / \mathbb{Z}\right) \cong C \otimes_{R} \tilde{F}$ for some flat $S$-module $\tilde{F}$. Thus by Lemma $3.4(2), H \otimes_{R} \tilde{F}$ is a $G_{\tilde{C}}$-flat $S$-module.

Corollary 4.14. Let $\varphi: R \rightarrow S$ be a homomorphism of rings with $f d_{R}(S)<\infty$. If $S$ is coherent, $H$ is a $G_{C}$-proj $R$-module and $\tilde{I}$ is an injective $S$-module, then $\operatorname{Hom}_{R}(H, \tilde{I})$ is a $G_{\tilde{C}}$-inj $S$-module, provided $F(S) \subseteq P(R)$.

Proof. Let $H$ be a $G_{C}$-proj $R$-module and $\tilde{I}$ an injective $S$-module. Under the assumption, $H \otimes_{R} S$ is a $G_{\tilde{C}}$-flat $S$-module by Proposition 4.13. Then $\operatorname{Hom}_{R}(H, \tilde{I}) \cong \operatorname{Hom}_{S}\left(H \otimes_{R} S, \tilde{I}\right)$ is a $G_{\tilde{C}}$-inj $S$-module by Proposition 4.2.

Proposition 4.15. Let $\varphi: R \rightarrow S$ be a homomorphism of Noetherian rings with $f d_{R}(S)<\infty$. If $H$ is a finitely generated $G_{C}$-proj $R$-module and $\tilde{I}$ is an injective $S$-module, then $\operatorname{Hom}_{R}(H, \tilde{I})$ is a $G_{\tilde{C}^{-}}$-inj $S$-module.

Proof. Let $\tilde{I}$ be an injective $S$-module. From Proposition 3.8 and its proof, we know that $H$ is $G_{C}$-flat and there exists a complete $P_{C}$-resolution $\mathbb{P}$ of $H$, which each $P_{i}$ and $P^{i}$ is finitely generated projective. Then $\mathbb{P}$ is also a complete $F_{C}$-resolution of $H$ by Lemma 3.6. Firstly, we have the following isomorphism:

$$
\operatorname{Hom}_{R}(\mathbb{P}, \tilde{I}) \cong \operatorname{Hom}_{S}\left(\mathbb{P} \otimes_{R} S, \tilde{I}\right) .
$$

Thus, $\operatorname{Hom}_{R}(\mathbb{P}, \tilde{I})$ is exact by Lemma 3.4(1). Secondly, by Proposition 3.3(3) we only need to prove that, for any injective $S$-module $\tilde{J}, \operatorname{Hom}_{S}\left(\operatorname{Hom}_{S}(\tilde{C}, \tilde{J})\right.$, $\left.\operatorname{Hom}_{R}(\mathbb{P}, \tilde{I})\right)$ is exact. In fact,

$$
\begin{aligned}
\operatorname{Hom}_{S}\left(\operatorname{Hom}_{S}(\tilde{C}, \tilde{J}), \operatorname{Hom}_{R}(\mathbb{P}, \tilde{I})\right) & \cong \operatorname{Hom}_{S}\left(\operatorname{Hom}_{S}(\tilde{C}, \tilde{J}), \operatorname{Hom}_{S}\left(\mathbb{P} \otimes_{R} S, \tilde{I}\right)\right) \\
& \cong \operatorname{Hom}_{S}\left(\operatorname{Hom}_{S}(\tilde{C}, \tilde{J}) \otimes_{R} \mathbb{P}, \tilde{I}\right)
\end{aligned}
$$




$$
\cong \operatorname{Hom}_{S}\left(\operatorname{Hom}_{R}(C, \tilde{J}) \otimes_{R} \mathbb{P}, \tilde{I}\right)
$$

is exact by Lemma $3.4(6)$. Hence, $\operatorname{Hom}_{R}(H, \tilde{I})$ is a $G_{\tilde{C}}$-inj $S$-module.

Proposition 4.16. Let $\varphi: R \rightarrow S$ be a homomorphism of rings with $f d_{R}(S)<$ $\infty$. If $B$ is a $G_{C}$-inj $R$-module and $\tilde{P}$ is a projective $S$-module, then $\operatorname{Hom}_{R}(\tilde{P}$, $B)$ is a $G_{\tilde{C}}$-inj $S$-module, provided $P(S) \subseteq P(R)$.

Proof. Let $\mathbb{I}$ be a complete $I_{C}$-resolution of a $G_{C}$-inj $R$-module $B$ and $\tilde{P}$ a projective $S$-module. Then, by Proposition 3.3(4) and Lemma 3.4(3) we only need to prove that, for any injective $S$-module $\tilde{J}, \operatorname{Hom}_{S}\left(\operatorname{Hom}_{S}(\tilde{C}, \tilde{J}), \operatorname{Hom}_{R}(\tilde{P}, \mathbb{I})\right)$ is exact. In fact,

$$
\begin{aligned}
\operatorname{Hom}_{S}\left(\operatorname{Hom}_{S}(\tilde{C}, \tilde{J}), \operatorname{Hom}_{R}(\tilde{P}, \mathbb{I})\right) & \cong \operatorname{Hom}_{R}\left(\operatorname{Hom}_{S}(\tilde{C}, \tilde{J}) \otimes_{S} \tilde{P}, \mathbb{I}\right) \\
& \cong \operatorname{Hom}_{R}\left(\operatorname{Hom}_{R}(C, \tilde{J}) \otimes_{S} \tilde{P}, \mathbb{I}\right) \\
& \cong \operatorname{Hom}_{S}\left(\tilde{P}, \operatorname{Hom}_{R}\left(\operatorname{Hom}_{R}(C, \tilde{J}), \mathbb{I}\right)\right)
\end{aligned}
$$

is exact by Lemma 3.4(4). Hence, $\operatorname{Hom}_{R}(\tilde{P}, B)$ is a $G_{\tilde{C}}$-inj $S$-module.

Corollary 4.17. Let $\varphi: R \rightarrow S$ be a homomorphism of rings with $f d_{R}(S)<\infty$. If $S$ is coherent, $B$ is a $G_{C}$-inj $R$-module and $\tilde{F}$ is a flat $S$-module, then $\operatorname{Hom}_{R}(\tilde{F}, B)$ is a $G_{\tilde{C}}$-inj $S$-module, provided $F(S)=P(S) \subseteq P(R)$.

Proof. Let $B$ be a $G_{C}$-inj $R$-module and $\tilde{F}$ a flat $S$-module. Consider the isomorphism $\operatorname{Hom}_{R}(\tilde{F}, B) \cong \operatorname{Hom}_{S}\left(\tilde{F}, \operatorname{Hom}_{R}(S, B)\right)$. Since $P(S) \subseteq P(R)$, the module $\operatorname{Hom}_{R}(S, B)$ is a $G_{\tilde{C}}$-inj $S$-module by Proposition 4.16, and since $F(S)=P(S)$, it follows by Theorem 4.4 applied to $\varphi=1_{R}$ that $\operatorname{Hom}_{S}(\tilde{F}$, $\left.\operatorname{Hom}_{R}(S, B)\right)$ is a $G_{\tilde{C}^{-}}$-inj $S$-module.

Let $S$ be a multiplicatively closed set of $R$. By Lemma 3.2, we know that $S^{-1} C$ is a semidualizing $S^{-1} R$-module. On the other hand, since $S^{-1} R$ is a flat $R$-module, each flat $S^{-1} R$-module is also a flat $R$-module and each injective $S^{-1} R$-module is also an injective $R$-module by [1, Corollary 4.2(b)].

Lemma 4.18 ([14, Lemma 3.16]). Let $S$ be a multiplicatively closed set of $R$. If $S^{-1} R$ is a projective $R$-module, then $\bar{A}$ is a projective $R$-module if and only if $\bar{A}$ is a projective $S^{-1} R$-module for any $S^{-1} R$-module $\bar{A}$.

Proposition 4.19. Let $S$ be a multiplicatively closed set of $R$. If $S^{-1} R$ is a projective $R$-module and $M$ is a $G_{C}$-proj $R$-module, then $S^{-1} M$ is a $G_{S^{-1} C^{-}}$ proj $S^{-1} R$-module.

Proof. Let the exact sequence of $R$-modules $\mathbb{X}=\cdots \rightarrow P_{1} \rightarrow P_{0} \rightarrow C \otimes_{R} Q^{0} \rightarrow$ $C \otimes_{R} Q^{1} \rightarrow \cdots$, where each $P_{i}$ and $Q^{i}$ is projective, be a complete $P_{C}$-resolution of $M$. Then the sequence of $S^{-1} R$-modules $S^{-1} \mathbb{X}=\cdots \rightarrow S^{-1} P_{1} \rightarrow S^{-1} P_{0} \rightarrow$ $S^{-1} C \otimes_{S^{-1} R} S^{-1} Q^{0} \rightarrow S^{-1} C \otimes_{S^{-1} R} S^{-1} Q^{1} \rightarrow \cdots$ is exact and each $S^{-1} P_{i}$ 
and $S^{-1} Q^{i}$ is projective. We now only need to prove that, for any projective $S^{-1} R$-module $\bar{Q}$,

$\operatorname{Ext}_{S^{-1} R}^{1}\left(S^{-1} K^{t}, S^{-1} C \otimes_{S^{-1} R} \bar{Q}\right)=0$ and $\operatorname{Ext}_{S^{-1} R}^{1}\left(S^{-1} K_{j}, S^{-1} C \otimes_{S^{-1} R} \bar{Q}\right)=0$, where $K^{t}$ and $K_{j}$ are cokernels of $\mathbb{X}$ for all $t \geqslant 0, j \geqslant 0$. In fact,

$$
\begin{aligned}
\operatorname{Ext}_{S^{-1} R}^{1}\left(S^{-1} K^{t}, S^{-1} C \otimes_{S^{-1} R} \bar{Q}\right) & \cong \operatorname{Ext}_{S^{-1} R}^{1}\left(S^{-1} R \otimes_{R} K^{t}, S^{-1} C \otimes_{S^{-1} R} \bar{Q}\right) \\
& \cong \operatorname{Ext}_{R}^{1}\left(K^{t}, C \otimes_{R} \bar{Q}\right)=0
\end{aligned}
$$

by [12, p. 258, 9.21], [11, Proposition 5.17] and Lemma 4.18. Similar for each $K_{j}$. This completes our proof.

Proposition 4.20. Let $S$ be a multiplicatively closed set of $R$. If $S^{-1} R$ is a projective $R$-module and $\bar{N}$ is a $G_{S^{-1} C^{-i n j}} S^{-1} R$-module, then $\bar{N}$ is a $G_{C}$-inj $R$-module.

Proof. Let the exact sequence of $S^{-1} R$-modules $\overline{\mathbb{Y}}=\cdots \rightarrow \operatorname{Hom}_{S^{-1} R}\left(S^{-1} C, \bar{I}^{1}\right)$ $\rightarrow \operatorname{Hom}_{S^{-1} R}\left(S^{-1} C, \overline{I^{0}}\right) \rightarrow \bar{I}_{0} \rightarrow \bar{I}_{1} \rightarrow \cdots$, where each $\bar{I}_{i}$ and $\bar{I}^{i}$ is injective, be a complete $I_{S^{-1} C^{-r e s o l u t i o n}}$ of $\bar{N}$. Since $S^{-1} R$ is a flat $R$-module, each $\bar{I}_{i}$ and $\bar{I}^{i}$ is also an injective $R$-module. We can also get that each $\operatorname{Hom}_{S^{-1} R}\left(S^{-1} C, \bar{I}^{i}\right)$ is a $C$-injective $R$-module, since $\operatorname{Hom}_{S^{-1} R}\left(S^{-1} C, \bar{I}^{i}\right) \cong \operatorname{Hom}_{S^{-1} R}\left(S^{-1} R \otimes_{R} C\right.$, $\left.\bar{I}^{i}\right) \cong \operatorname{Hom}_{R}\left(C, \bar{I}^{i}\right)$. We now only need to prove that, for any injective $R$-module $E$,

$$
\operatorname{Ext}_{R}^{1}\left(\operatorname{Hom}_{R}(C, E), \bar{K}^{t}\right)=0 \text { and } \operatorname{Ext}_{R}^{1}\left(\operatorname{Hom}_{R}(C, E), \bar{K}_{j}\right)=0,
$$

where $\bar{K}^{t}$ and $\bar{K}_{j}$ are cokernels of $\overline{\mathbb{Y}}$ for all $t \geqslant 0, j \geqslant 0$. In fact,

$$
\begin{aligned}
\operatorname{Ext}_{R}^{1}\left(\operatorname{Hom}_{R}(C, E), \bar{K}^{t}\right) & \cong \operatorname{Ext}_{S^{-1} R}^{1}\left(\operatorname{Hom}_{R}(C, E) \otimes_{R} S^{-1} R, \bar{K}^{t}\right) \\
& \cong \operatorname{Ext}_{S^{-1} R}^{1}\left(\operatorname{Hom}_{S^{-1} R}\left(S^{-1} C, S^{-1} E\right), \bar{K}^{t}\right)=0
\end{aligned}
$$

by $\left[12\right.$, p. 258, 9.21] and [11, Proposition 5.17]. Similar for each $\bar{K}_{j}$. This completes our proof.

Proposition 4.21. Let $S$ be a multiplicatively closed set of $R$. Then the following hold:

(1) If $M$ is a $G_{C}$-flat $R$-module, then $S^{-1} M$ is a $G_{C}$-flat $R$-module for any $R$-module $M$.

(2) If $M$ is a $G_{C}$-flat $R$-module, then $S^{-1} M$ is a $G_{S^{-1} C^{-f l a t}} S^{-1} R$-module for any $R$-module $M$.

(3) For any $S^{-1} R$-module $\bar{M}, \bar{M}$ is a $G_{C}$-flat $R$-module if and only if $\bar{M}$ is a $G_{S^{-1} C}$-flat $S^{-1} R$-module.

Proof. (1) Let the exact sequence of $R$-modules $\mathbb{Z}=\cdots \rightarrow F_{1} \rightarrow F_{0} \rightarrow$ $C \otimes_{R} F^{0} \rightarrow C \otimes_{R} F^{1} \rightarrow \cdots$, where each $F_{i}$ and $F^{i}$ is flat, be a complete $F_{C^{-}}$ resolution of $M$. Then the sequence of $S^{-1} R$-modules $S^{-1} \mathbb{Z}=\cdots \rightarrow S^{-1} F_{1} \rightarrow$ $S^{-1} F_{0} \rightarrow S^{-1} C \otimes_{S^{-1} R} S^{-1} F^{0} \rightarrow S^{-1} C \otimes_{S^{-1} R} S^{-1} F^{1} \rightarrow \cdots$ is exact and each $S^{-1} F_{i}$ and $S^{-1} F^{i}$ is flat. Since $S^{-1} R$ is a flat $R$-module, each $S^{-1} F_{i}$ and $S^{-1} F^{i}$ 
is also a flat $R$-module. We also know that $S^{-1} C \otimes_{S^{-1} R} S^{-1} F^{i} \cong C \otimes_{R} S^{-1} F^{i}$ by [11, Proposition 5.17] is a $C$-flat $R$-module for all $i \geqslant 0$.

On the other hand, let $I$ be an injective $R$-module. Then

$$
\operatorname{Hom}_{R}(C, I) \otimes_{R} S^{-1} \mathbb{Z} \cong S^{-1}\left(\operatorname{Hom}_{R}(C, I) \otimes_{R} \mathbb{Z}\right)
$$

is exact by [11, Proposition 5.17]. Hence, $S^{-1} M$ is a $G_{C}$-flat $R$-module.

(2) Let $\bar{I}$ be an injective $S^{-1} R$-module and $K^{t}$ and $K_{j}$ cokernels of $\mathbb{Z}$ for all $t \geqslant 0, j \geqslant 0$. Then $\operatorname{Tor}_{1}^{S^{-1} R}\left(\operatorname{Hom}_{S^{-1} R}\left(S^{-1} C, \bar{I}\right), S^{-1} K^{t}\right) \cong \operatorname{Tor}_{1}^{R}\left(\operatorname{Hom}_{R}(C, \bar{I})\right.$, $\left.K^{t}\right) \otimes_{R} S^{-1} R=0$ by [11, Proposition 5.17] and $\bar{I}$ is also an injective $R$-module. Similarly for each $K_{j}$. Then we get our goal.

$(3)(\Rightarrow)$ By $(2)$.

$(\Leftarrow)$ Let the exact sequence of $S^{-1} R$-modules $\overline{\mathbb{K}}=\cdots \rightarrow \bar{F}_{1} \rightarrow \bar{F}_{0} \rightarrow$ $S^{-1} C \otimes_{S^{-1} R} \bar{F}^{0} \rightarrow S^{-1} C \otimes_{S^{-1} R} \bar{F}^{1} \rightarrow \cdots$, where each $\bar{F}_{i}$ and $\bar{F}^{i}$ is flat, be

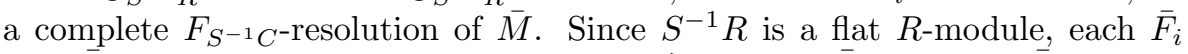
and $\bar{F}^{i}$ is also a flat $R$-module. From $S^{-1} C \otimes_{S^{-1} R_{-}} \bar{F}^{i} \cong C \otimes_{R} \bar{F}^{i}$ by [11, Proposition 5.17], we know that each $S^{-1} C \otimes_{S^{-1} R} \bar{F}^{i}$ is a $C$-flat $R$-module. On the other hand, let $I$ be an injective $R$-module. Then $\operatorname{Hom}_{R}(C, I) \otimes_{R} \overline{\mathbb{K}} \cong$ $\operatorname{Hom}_{S^{-1} R}\left(S^{-1} C, S^{-1} I\right) \otimes_{S^{-1} R} \overline{\mathbb{K}}$ is exact by [11, Proposition 5.17]. Hence, $\bar{M}$ is a $G_{C}$-flat $R$-module.

Acknowledgments. The authors would like to thank the referee for helpful suggestions and corrections.

\section{References}

[1] L. L. Avramov and H. B. Foxby, Homological dimensions of unbounded complexes, J. Pure Appl. Algebra. 71 (1991), no. 2-3, 129-155.

[2] L. W. Christensen, Gorenstein Dimensions, Lecture Notes in Mathematics, vol. 1747, Springer-Verlag, Berlin, 2000.

[3] L. W. Christensen and H. Holm, Ascent properties of Auslander categories, Canad. J. Math. 61 (2009), no. 13, 76-108.

[4] E. E. Enochs and O. M. G. Jenda, Relative Homological Algebra, Walter de Gruyter, New York, Spring-Verlag, 2000.

[5] H. B. Foxby, Gorenstein modules and related modules, Math. Scand. 31 (1972), 267-284.

[6] E. S. Golod, G-dimension and generalized perfect ideals, Trudy Mat. Inst. Steklov. 165 (1984), 62-66.

[7] H. Holm, Gorenstein homological dimensions, J. Pure Appl. Algebra. 189 (2004), no. $1-3,167-193$

[8] H. Holm and P. Jøgensen, Semi-dualizing modules and related Gorenstein homological dimensions, J. Pure Appl. Algebra. 205 (2006), no. 2, 423-445.

[9] H. Holm and D. White, Foxby equivalence over associative rings, J. Math. Kyoto Univ. 47 (2007), no. 4, 781-808.

[10] T. Y. Lam, Lecture on Modules and Rings, Springer-Verlag, New York, 1999.

[11] M. S. Osborne, Basic Homological Algebra, Graduate Texts in Mathematics, 196. Springer-Verlag, New York, 2000.

[12] J. J. Rotman, An Introduction to Homological Algebra, Academic Press, New York, 1979.

[13] W. V. Vasconcelos, Divisor Theory in Module Categories, North-Holland Publishing Co., Amsterdam, 1974. 
[14] X. Y. Yang and Z. K. Liu, Strongly Gorenstein projective, injective and flat modules, J. Algebra. 320 (2008), no. 7, 2659-2674.

[15] D. White, Gorenstein projective dimension with respect to a semidualizing module, J. Commut. Algebra 2 (2010), no. 1, 111-137.

ZhenXing Di

Department of Mathematics

SOUTHEAST UNIVERSITY

Nanjing 210096, Jiangsu, P. R. China

E-mail address: dizhenxing19841111@126.com

\section{XiaOYAN YANG}

College of Mathematics and Information Science

Northwest Normal University

Lanzhou 730070, Gansu, P. R. China

E-mail address: yxy800218@163.com 